\title{
ONE-SIDED WIDTHS OF CLASSES OF SMOOTH FUNCTIONS $^{1}$
}

\author{
Yurii N.Subbotin
}

N.N. Krasovskii Institute of Mathematics and Mechanics of the Ural Branch of the Russian Academy of Sciences and Ural Federal University, Ekaterinburg, Russia, yunsub@imm.uran.ru

\footnotetext{
One-sided widths of the classes of functions $W_{p}^{r}[0,1]$ in the metric $L_{q}[0,1], 1 \leq p, q \leq \infty, r \geq 1$, are studied. Such widths are defined similarly to Kolmogorov widths with additional constraints on the approximating functions.

Keywords: One-sided widths, Exact orders, Classes of smooth functions.
}

Let us introduce some definitions. The Kolmogorov width (see [1]) is, by definition, the value

$$
d_{n}\left(W_{p}^{r}, L_{q}\right)=\inf _{L_{n} \subset L_{q}} \sup _{f \in W_{p}^{r}} \inf _{g(x) \in L_{n}}\|f-g\|_{L_{q}},
$$

where $L_{n}$ is an $n$-dimensional subspace of the space $L_{q}[0,1] ; W_{p}^{r}$ is the class of functions $f(x)$ representable in the form

$$
f(x)=P_{r-1}(x)+\frac{1}{(r-1) !} \int_{0}^{x}(x-t)^{r-1} f^{(r)}(t) d t .
$$

Here, $P_{r-1}(x)$ is a polynomial of degree at most $r-1, r$ is a positive integer, and $r \geq 1 ; f^{(r-1)}(x)$ is absolutely continuous and $\left\|f^{(r)}\right\|_{L_{p}}=\left(\int_{0}^{1}\left|f^{(r)}(x)\right|^{p} d x\right)^{1 / p} \leq 1,1 \leq p \leq \infty$; by $\left\|f^{(r)}\right\|_{L_{\infty}}$ we mean $\operatorname{ess} \sup \left\{\left|f^{(r)}(x)\right|: 0 \leq x \leq 1\right\}$.

The corresponding one-sided width is defined as follows (see [2]):

$$
d_{n}^{+}\left(W_{p}^{r}, L_{q}\right)=\inf _{L_{n} \subset L_{q}} \sup _{f \in W_{p}^{r}} \inf _{\substack{g(x) \in L_{n} \\ g(x) \geq f(x)}}\|f-g\|_{L_{q}} .
$$

Orders of widths $d_{n}\left(W_{p}^{r}, L_{q}\right)$ (1) with respect to $n$ were studied by many authors. Detailed information on this subject is given quite completely in [3], where the final results in this direction were obtained. The following final order result is valid:

$$
d_{n}\left(W_{p}^{r}, L_{q}\right) \asymp\left\{\begin{array}{cl}
n^{-r}, & \text { if } 1 \leq q \leq p \leq \infty \quad \text { or } \quad 2<p \leq q \leq \infty, \\
n^{-r-\frac{1}{2}+\frac{1}{p}}, & \text { if } 1 \leq p \leq 2 \leq q \leq \infty \\
n^{-r-\frac{1}{q}+\frac{1}{p}}, & \text { if } 1 \leq p<q \leq 2
\end{array}\right.
$$

where the symbol $\asymp$ means that the upper and lower bounds hold for $d_{n}\left(W_{p}^{r}, L_{q}\right)$ with the given orders with respect to $n$ accurately to the constants that depend only on $r, p$ and $q$.

In the present paper, we show that one-sided widths $d_{n}^{+}\left(W_{p}^{r}, L_{q}\right)$ have the same orders (2) with respect to $n$.

\footnotetext{
${ }^{1}$ Published in Russian in Trudy Inst. Mat. i Mekh. UrO RAN, 2012. Vol. 18. No 4. P. 267-270.
} 
Theorem. For all positive integers $r \geq 1$ and $1 \leq p, q \leq \infty$, the following order equalities are valid:

$$
d_{n}^{+}\left(W_{p}^{r}, L_{q}\right) \asymp\left\{\begin{array}{cl}
n^{-r}, & \text { if } 1 \leq q \leq p \leq \infty \quad \text { or } \quad 2<p \leq q \leq \infty, \\
n^{-r-\frac{1}{2}+\frac{1}{p}}, & \text { if } 1 \leq p \leq 2 \leq q \leq \infty \\
n^{-r-\frac{1}{q}+\frac{1}{p}}, & \text { if } 1 \leq p<q \leq 2 .
\end{array}\right.
$$

P r o o f. Since, by definition, $d_{n}^{+}\left(W_{p}^{r}, L_{q}\right) \geq d_{n}\left(W_{p}^{r}, L_{q}\right)$ and (2) is valid, the lower bounds follow immediately.

Estimating the widths from above, we consider several cases. Divide the interval $[0,1]$ into $n$ equal intervals $\left[x_{i}, x_{i+1}\right](i=0,1, \ldots, n-1), x_{i}=i / n$. On each interval, we will approximate a function $f(x)$ from $W_{p}^{r}$ by the Taylor partial sum

$$
\varphi_{i, r}(x)=f\left(\bar{x}_{i}\right)\left(x-\bar{x}_{i}\right)+\cdots+f^{(r-1)}\left(\bar{x}_{i}\right) \frac{\left(x-\bar{x}_{i}\right)^{r-1}}{(r-1) !}, \quad \bar{x}_{i}=\frac{x_{i}+x_{i+1}}{2} .
$$

We have

$$
\left|f(x)-\varphi_{i, r}(x)\right|=\left|\frac{1}{(r-1) !} \int_{\bar{x}_{i}}^{x}(x-t)^{r-1} f^{(r)}(t) d t\right|, \quad x \in\left[x_{i}, x_{i+1}\right] .
$$

The following estimates hold $\left(1 / p+1 / p_{1}=1\right)$ :

$$
\begin{gathered}
\left|f(x)-\varphi_{i, r}(x)\right| \leq \frac{1}{(r-1) !}\left|\int_{\bar{x}_{i}}^{x}(x-t)^{r-1} f^{(r)}(t) d t\right| \\
\leq \frac{1}{(r-1) !}\left|\int_{\bar{x}_{i}}^{x}\right| x-\left.\left.\left.\left.t\right|^{(r-1) p_{1}} d t\right|^{\frac{1}{p_{1}}}\left|\int_{\bar{x}_{i}}^{x}\right| f^{(r)}(t)\right|^{p} d t\right|^{\frac{1}{p}} \\
\leq \frac{1}{(r-1) !}\left|x-\bar{x}_{i}\right|^{\frac{(r-1) p_{1}+1}{p_{1}}}\left(\int_{x_{i}}^{x_{i+1}}\left|f^{(r)}(t)\right|^{p} d t\right)^{\frac{1}{p}} \\
\leq \frac{1}{(r-1) !} \frac{\left(x_{i+1}-x_{i}\right)^{r-1+\frac{1}{p_{1}}}}{2^{r-1+\frac{1}{p_{1}}}}\left(\int_{x_{i}}^{x_{i+1}}\left|f^{(r)}(t)\right|^{p} d t\right)^{\frac{1}{p}}=C_{i} .
\end{gathered}
$$

Thus, the following inequalities are valid:

$$
\begin{gathered}
f(x)-\varphi_{i, r}(x)+C_{i} \geq 0 \quad(i=0,1, \ldots, n-1), \\
0 \leq f(x)-\varphi_{i, r}(x)+C_{i} \leq 2 C_{i}=\frac{1}{(r-1) !} \frac{\left(x_{i+1}-x_{i}\right)^{r-1+\frac{1}{p_{1}}}}{2^{r+\frac{1}{p_{1}}}}\left(\int_{x_{i}}^{x_{i+1}}\left|f^{(r)}(t)\right|^{p} d t\right)^{\frac{1}{p}} .
\end{gathered}
$$

Denote by $L_{n r}$ the $n r$-dimensional subspace of functions $g(x)$ of the form

$$
g(x)=P_{r-1, i}(x), \quad x \in\left[x_{i}, x_{i+1}\right] \quad(i=0,1, \ldots, n-1),
$$

where $P_{r-1, i}(x)$ is a polynomial of degree at most $r-1$. Then, for the functions from (3)-(6), which belong to $L_{n r}$, we have

$$
d_{n r_{1}}^{+}\left(W_{p}^{r}, L_{q}\right) \leq\left(\sum_{i=0}^{n-1} \int_{x_{i}}^{x_{i+1}}\left|f(x)-\varphi_{i, r}(x)+C_{i}\right|^{q} d x\right)^{\frac{1}{q}}
$$




$$
\leq\left[\sum_{i=0}^{n-1}\left(x_{i+1}-x_{i}\right)\left(2 C_{i}\right)^{q}\right]^{\frac{1}{q}} \leq\left(\frac{1}{n}\right)^{r-\frac{1}{p}+\frac{1}{q}} \frac{1}{(r-1) ! 2^{r-\frac{1}{p}}}\left[\sum_{i=0}^{n-1}\left(\int_{x_{i}}^{x_{i+1}}\left|f^{(r)}(t)\right|^{p} d t\right)^{\frac{q}{p}}\right]^{\frac{1}{q}} .
$$

Denote $\alpha_{i}=\int_{x_{i}}^{x_{i+1}}\left|f^{(r)}(t)\right|^{p} d t \geq 0$. Since $f \in W_{p}^{r}$, we have $\sum_{i=0}^{n-1} \alpha_{i}=1$. This and (7) imply that $\sum_{i=0}^{n-1} \alpha_{i}^{\frac{q}{p}}$ achieves the largest value for $q / p>1$ if one of $\alpha_{i}$ is equal to 1 and all the other are zero; i. e., in this case,

$$
d_{n r}^{+}\left(W_{p}^{r}, L_{q}\right) \leq \frac{1}{(r-1) ! 2^{r-\frac{1}{p}}}\left(\frac{1}{n}\right)^{r-\frac{1}{p}+\frac{1}{q}}, \quad q>p .
$$

For $q \leq p$, the largest value on the right-hand side of $(7)$ is achieved for $\alpha_{i}=(1 / n)$; i. e., in this case,

$$
\begin{gathered}
d_{n r}^{+}\left(W_{p}^{r}, L_{q}\right) \leq \frac{1}{(r-1) ! 2^{r-\frac{1}{p}}}\left(\frac{1}{n}\right)^{r-\frac{1}{p}+\frac{1}{q}}\left[\sum_{i=0}^{n-1}\left(\frac{1}{n}\right)^{\frac{q}{p}}\right]^{\frac{1}{q}} \\
=\frac{1}{(r-1) ! 2^{r-\frac{1}{p}}}\left(\frac{1}{n}\right)^{r-\frac{1}{p}+\frac{1}{q}}\left(\frac{1}{n}\right)^{\frac{1}{p}} n^{\frac{1}{q}}=\frac{1}{(r-1) ! 2^{r-\frac{1}{p}}}\left(\frac{1}{n}\right)^{r} \quad(q \leq p) .
\end{gathered}
$$

Further, consider the case $2<p \leq q \leq \infty$. Here, we use a fact mentioned in [3]. The following inequalities are valid:

$$
d_{n}^{+}\left(W_{p}^{r}, L_{q}\right) \leq d_{n}^{+}\left(W_{p}^{r}, L_{\infty}\right) \leq d_{n}^{+}\left(W_{2}^{r}, L_{\infty}\right) .
$$

The former inequality in (9) follows from the inequality $\|f\|_{L_{q}} \leq\|f\|_{L_{\infty}}$, and the latter inequality follows from the embedding $W_{p}^{r} \subset W_{2}^{r}$ because

$$
\left(\int_{0}^{1}\left|f^{(r)}(x)\right|^{2} d x\right)^{\frac{1}{2}} \leq\left(\int_{0}^{1}\left|f^{(r)}(x)\right|^{2 \cdot \frac{p}{2}} d x\right)^{\frac{1}{p}}\left(\int_{0}^{1}(1)^{\frac{p}{p-2}} d x\right)^{\frac{p-2}{p}}=\left(\int_{0}^{1}\left|f^{(r)}(x)\right|^{p} d x\right)^{\frac{1}{p}} .
$$

From inequality (9) for $2<p \leq q \leq \infty$, we deduce that

$$
\begin{gathered}
d_{n}\left(W_{p}^{r}, L_{q}\right) \leq d_{n}^{+}\left(W_{p}^{r}, L_{q}\right) \leq d_{n}^{+}\left(W_{2}^{r}, L_{\infty}\right) \leq 2 d_{n}\left(W_{2}^{r}, L_{\infty}\right) \asymp n^{-r}, \\
2<p \leq q \leq \infty ;
\end{gathered}
$$

i. e., in this case,

$$
d_{n}^{+}\left(W_{2}^{r}, L_{\infty}\right) \asymp n^{-r}, \quad 2 \leq p \leq q \leq \infty .
$$

It remains to prove that $d_{n}^{+}\left(W_{p}^{r}, L_{q}\right) \asymp n^{-r-\frac{1}{2}+\frac{1}{p}}$ for $1 \leq p \leq 2 \leq q \leq \infty$. Taking into account the former inequality in (9), we have

$$
d_{n}^{+}\left(W_{p}^{r}, L_{q}\right) \leq d_{n}^{+}\left(W_{p}^{r}, L_{\infty}\right)
$$

Note the following fact. If a set $W[0,1]$ from $L_{\infty}$ contains an arbitrary constant, then approximating subspaces must also contain this constant. Otherwise, $d_{n}\left(W, L_{\infty}\right)=\infty$. Therefore,

$$
d_{n}\left(W_{p}^{r}, L_{\infty}\right) \leq d_{n}^{+}\left(W_{p}^{r}, L_{\infty}\right)=\inf _{L_{n}} \sup _{f \in W_{p}^{r}} \inf _{\substack{g(x) \in L_{n} \\ g(x) \geq f(x)}}\|f-g\|_{L_{q}}
$$

$\leq \inf _{L_{n}} \sup _{f \in W_{p}^{r}} \inf _{g(x) \in L_{n}}\left\|f-g+d_{n}\left(W_{p}^{r}, L_{\infty}\right)\right\|_{L_{\infty}} \leq 2 d_{n}\left(W_{p}^{r}, L_{\infty}\right) \asymp n^{-r+\frac{1}{2}+\frac{1}{p}} \quad(1 \leq p \leq 2 \leq q \leq \infty)$.

For the latter equivalence, see the case $p \leq 2 \leq q \leq \infty$ in (2). 
For a given $m$, we find $[m / r]$, where $[m / r]$ is the integer part of the number $m / r$. Then, $[m / r] r \leq m \leq([m / r]+1) r$. In this case,

$$
d_{\left[\frac{m}{r}\right] r+1}^{+}\left(W_{p}^{r}, L_{q}\right) \leq d_{m}^{+}\left(W_{p}^{r}, L_{q}\right) \leq d_{\left[\frac{m}{r}\right] r}^{+}\left(W_{p}^{r}, L_{q}\right)
$$

and, from the foregoing, we obtain the exact order of behavior of the one-sided widths with respect to $m(m \rightarrow \infty)$ for all $m$, not only for $m$ that are multiples of $r$. Moreover, the equivalence constants are finite and depend only on $r, p$, and $q ; r \in \mathbb{N}$ and $1 \leq p, q \leq \infty$.

For an even positive integer $r$, we can also use the results from [4]. Then, in a number of cases, estimating from above, we can obtain the constants independent of $n$ that may be less than the constants in the present paper; however, the order of their behavior with respect to $n(n \rightarrow \infty)$ will be the same.

\section{REFERENCES}

1. Kolmogoroff A. Über die beste Annäherung von Funktionen einer geqebenen Functionenklasse // Ann. Math. 1936. Vol. 37. P. 107-111.

2. Korneichuk N.P., Ligun A.A. and Doronin V.G. Approximation With Constraints Kiev: Naukova Dumka, 1982. 250 p. [in Russian].

3. Kashin B.S. Diameters of some finite-dimensional sets and classes of smooth functions // Izv. Math. 1977. Vol. 11, no. 2. P. 334-351.

4. Birkhoff G., Schultz M.H. and Varga R.S. Piecewise Hermite interpolation in one and two variables with application to partial differential equations // Numer. Math. 1968. Vol. 11. P. 232-256. 